\title{
Application of Reinforcement Learning as a Tool of Adaptive Traffic Signal Control on Isolated Intersections
}

\author{
Kristi Bombol, Daniela Koltovska, and Kostandina Veljanovska
}

\begin{abstract}
For a long time it was believed that the systems responding to real time traffic would enable significant benefits. However, numerous limitations have appeared such as the existence of the models with high level of detail precision, the uncertainty in predicting future traffic flows, the difficulty in arrival time estimation, the lack of self-adjusting mechanism.

The difficulties in optimising the signal control strategy have initiated new researches. The results highlight the artificial intelligence methods as a possible solution. These systems are characterized with the ability to accumulate and use knowledge, set a problem, learn, process, conclude, solve the problem and exchange knowledge.

The research presented in this paper proposes an adaptive signal control performed by a control agent able to adapt to an optimal policy by learning from the environment. The goal to be achieved is minimization of the delays in the system.

First, the problem of reinforcement learning has been set. The first computation results of the Q-learning application for adaptive traffic signal control are presented.

It is concluded that the results obtained are in favor of the adaptive signal control strategy compared to the fixed and actuated signal control.
\end{abstract}

Index Terms-Delay, isolated intersection, $\mathrm{Q}$ learning, simulations.

\section{INTRODUCTION}

The Intelligent Transport Systems (ITS) used by the advanced technologies in the transport system are an approach widely dispersed as a solution to the traffic problems that the society faces. The aim of the use of ITS is improvement of the traffic quality by avoiding traffic congestions, saving travelling time, improving the safety and the comfort of the drivers and the passengers, improving the transport and the exchange of goods and services, and improvement of the entire informative transparency. Once achieved, all of these will lead to a higher level of satisfaction of the user's needs and prosperity of the surrounding area. Yet, there are more areas in which ITS can be applied and the traffic management and control in cities make only one of them.

Traffic signal control strategies are the most commonly used for managing the traffic flow at different types of

Manuscript received February 19, 2012; revised March 31, 2012.

Kristi Bombol and Daniela Koltovska are with the Department of Traffic and Transport Faculty of Technical Sciences - Bitola University St. Kliment Ohridski - Bitola, Republic of Macedonia (e-mail: kristi.bombol@uklo.edu.mk, daniela.koltovska@uklo.edu.mk).

Kostandina Veljanovska is with the Information Systems Managemen Faculty of Administration and Information Systems Management - Bitola University St. Kliment Ohridski - Bitola, Republic of Macedonia (e-mail: kostandina@rocketmail.com). intersections in the cities. At a signalized intersection they operate in one of the three different control modes: pre-timed control, semi actuated control, and fully-actuated control (Wilshire et al., 1985) [7].

Over the past two decades, significant efforts have been made to develop efficient and practical real time strategies to control the traffic at intersections. Although the concept of these efforts is promising, there are still some difficulties. The need to predict the origin and destination traffic demand in real time, the inherent limits in modeling the complex traffic flows, and the lack of confident sensors impose further investigations [8].

The researchers are expected to find simpler methods. Those have to include directly measured traffic parameters in the determination of the number of vehicles allowed to pass (through traffic signals), without the prediction in real time. The artificial intelligence can offer a very different approach to solve the above mentioned problems. The artificial intelligent systems reach the same results that man does when performing cognitive tasks. These systems are characterized with the ability to accumulate and use knowledge, set a problem, learn, process, conclude, solve the problem, and exchange knowledge.

The machine learning is a field of artificial intelligence. The reinforcement learning is a technique within the machine learning. It is successfully applied in solving problems, e.g. operating the elevators and robot soccer games. It is also applied in modeling the supply chain, dynamic allocation of resources, predicting time series [3].

In this research the reinforcement learning was applied in development of adaptive signal control strategy at an isolated intersection.

The paper is divided into four parts. The theory of the reinforcement learning is presented in the first part. The system model, including definitions pertaining the state, action, and reward function are in the second part. Part 3 presents and discusses the simulation results. The conclusions follow in part 4.

The analysis of the results of the simulation showed significant improvements in comparison with those of the fixed and fully-actuated signal control.

\section{ARTIFICIAL INTELLIGENCE}

\section{A. Reinforcement Learning (RL) Theory}

The ability to learn is one of the characteristics that define intelligence. That is why machine learning is center point of the artificial intelligence. 
The reinforcement learning is a subfield of the machine learning, learning what to do i.e. how to map the state into actions, how to maximize the numerical reward, and in which way [1]. It is a type of learning driven by interaction with the environment and directed to a goal. It is a technique that does not need monitoring and it is different from the methods of the supervised learning (e.g. neural networks) [1] [2].

The learner or decision maker is named agent, and everything it interacts with is named environment. The agent has a set of sensors to observe the state of the environment, and to perform a set of actions in order to change the state of the environment. The most important characteristics of the agent are: trial and error search and delayed reward.

The learner or an autonomous agent that senses its environment or acts in it can learn through trials to select the optimal action or actions which lead to the highest reward.

For a more accurate presentation of the interaction we here assume that the agent and the environment communicate in each sequence of discrete time steps: $t=0,1,2, \ldots$ In each time step, $\mathrm{t}$, the agent receives some representation of the state of the environment, $s_{t} \in S$, where $S$ is the set of possible states. In accordance with that, action $a_{t} \in A\left(s_{t}\right)$ is chosen, where $A\left(s_{t}\right)$ is a set of actions which are available in the state $s_{t}$. One step later, as a consequence of its action, the agent gets a numerical reward, $r_{t+l} \in R$ and finds itself in a new state, $S_{t+1}$. The teacher provides a reward or a penalty in order to induce the desirability of the final state. Fig. 1 shows the agent-environment interaction.

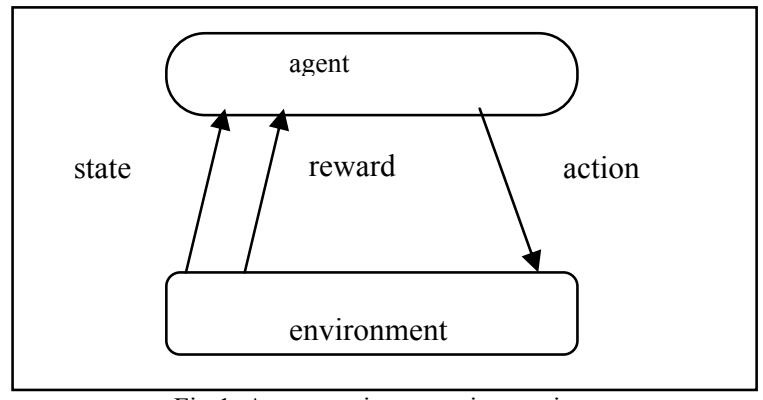

Fig 1. Agent-environment interaction.

The transition from one to another state is shown as:.

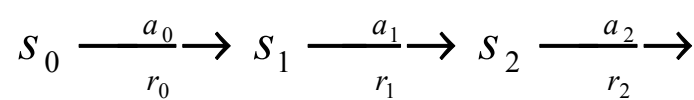

where

$S_{\mathrm{i}}$ is the state in the time step i,

$a_{\mathrm{i}}$ is the possible action available in each state in the time step i,

$r_{\mathrm{i}}$ is the reward which the agent receives in the time step $\mathrm{i}$ for taking action $a_{\mathrm{i}}$.

\section{B. Reinforcement Learning Elements}

The reinforcement learning has four basic elements, such as:

Policy - In each time step, the agent maps the presentation of the state with the probability of selection of each possible action. This mapping is called agent policy $\pi_{t}$, where $\pi_{t}(s, a)$ is the probability that $a_{t}=a$ if $s_{t}=s$. The reinforcement methods determine how the agent changes its policy as a result of its experience. The agent's goal, generally speaking, is to maximize the total reward it receives in long term.

Reward function - In the case of the reinforcement learning, the agent's goal is formalized in a sense of special signal, called reward, which is transmitted from the environment towards the agent. The reward is simply a number whose value differs from step to step. Informally, the agent's goal is to maximize the reward it receives. This means maximizing the reward that it receives in the moment as well as maximizing the cumulative reward that it receives in a long term.

Value function - Almost all reinforcement learning algorithms are based on the assessment of value functions state functions (or state-action pairs) which assess how good a given state is for the agent to be in (or how good some action is to conduct in a given state). The term 'how good' is defined here in a sense of future rewards that can be expected, or more precisely said, in a sense of expected earning (compensation). Of course, the rewards that the agent expects to receive in the future depend on the action it will conduct. Accordingly, the value functions are defined in accordance with some policies.

As the agent learns directly from the interaction with the real environment, it does not need the model of environment. The agent can be taught in advance by means of a simulator. The use of a simulator helps the agent to avoid the unacceptable and degrading actions in the field. In a case of real time traffic control, this type of action can have a counter effect on the system in particular.

\section{Adaptive Signal Control Strategy Design on ISOLATED INTERSECTION}

With the aim of collecting research data for this control strategy, simple network was created in a traffic micro simulator PTV Vision VISSIM COM (Fig. 2).

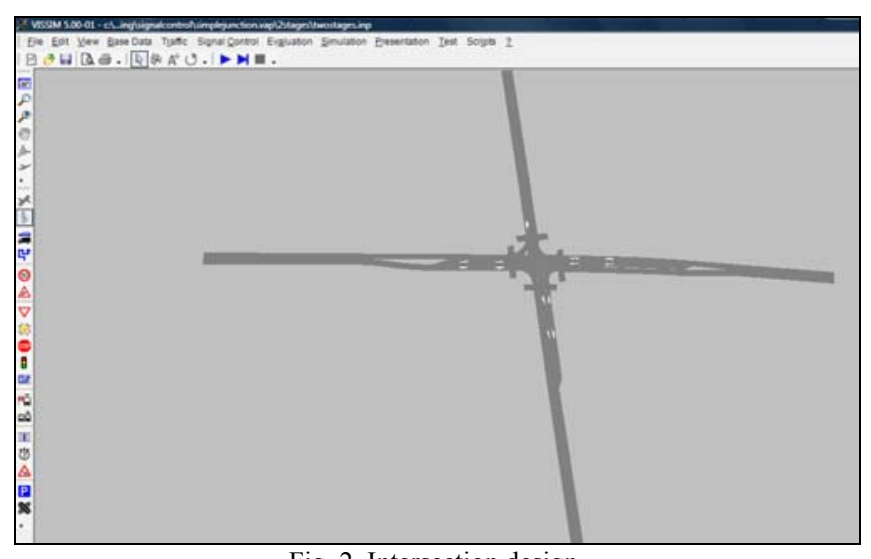

Fig. 2. Intersection design

The input data consisted of:

- Data on the traffic network - created graphically

- Data on the traffic demand - entered through the graphical interface of the simulator as a profile of traffic demand in all zones - origins of traffic.

The data for measuring the efficiency of the control strategy were collected with the assistance of the functions inside the VISSIM and then, saved separately.

The functions were called in each time step. The time step in use was 1 second. The program for communication with 
the simulator, the collecting of the data, and the reinforcement learning algorithm were done in VBA.

The design process was done in the following steps:

- Creation of traffic network with graphical implementation of the intersection, creation of links, connectors, traffic signals, setting detectors

- Creation of traffic demand

- Gathering and analysis of the results

As it can be seen in Fig.2, there are four zones for generation of traffic demand, one for each approach. The name, the position and the dimensions of the detector were defined with the help of the graphical interface of the simulator. Each detector was linked to appropriate traffic signal.

Powerful tool from the Intelligent transport systems that uses the technique of the artificial intelligence, so-called reinforcement learning - the Q-learning approach was chosen to deal with the stochastic nature of the traffic and to develop adaptive signal control strategy at the intersection.

\section{A. Definiton of the RL Elements for the Problem Researched}

The reinforcement learning elements were defined - states (s), actions (a), and rewards (r). The isolated intersection was two-phased and the number of states was eight with two possible actions each.

In the developing strategy for the intersection shown in Fig. 2 we set:

\section{States:}

[green][no gap][no occupancy]

[green][no gap][occupancy]

[green][gap][no occupancy]

[green][gap][occupancy]

[red][no gap][no occupancy]

[red][no gap][occupancy]

[red][gap][no occupancy]

[red][gap][occupancy]

Action set was defined as:

$0 \ldots$ take action to change the phase

1 ... take action to continue the green phase

The choice of action for this research was made according to $\varepsilon$ - greedy policy, where the best action is used with the possibility $1-\varepsilon$, and the research action was chosen by random choice with probability $\varepsilon$.

Reward was defined as a total number of vehicles at the intersection.

The Q-learning approach was chosen to deal with the stochastic nature of the traffic and to develop adaptive signal control strategy at the intersection. The Q-learning algorithm [1] (control algorithm of the temporary difference (off-policy TD Control)) is shown in its procedural form in the following way:

Initialize $Q(s, a)$ arbitrarily

Repeat (for each episode):

Initialize $s$

Repeat (for each step of episode): - greedy)

Choose $a$ from $s$ using policy derived from $Q$ (e.g.,

Take action $a$, observe $r, s^{\prime}$

$Q(s, a) \longleftarrow-Q(s, a)+\alpha\left[r+\gamma \max _{\alpha^{\prime}} Q\left(s^{\prime}, a^{\prime}\right)-Q(s, a)\right]$
$\mathrm{S} \leftarrow \mathrm{s}^{\prime}$

until $s$ is terminal.

In the algorithm above $Q(s, a)$ is the function of the action value, $\alpha$ is the learning percentage, $r$ is the reward, $\gamma$ is the discount rate, $Q\left(s^{\prime}, a^{\prime}\right)$ is the value function for the new action $a^{\prime}$ and the new state $s^{\prime}$. S is the set of possible states.

\section{Simulation Results}

In order to evaluate the proposed adaptive control strategy, the results obtained by the agent were compared with the results obtained by fixed and actuated signal control.

The testing was performed after sufficient number of iterations with different values of the conditions and after converging the Q-values. The number of iterations needed for convergence depends on the size of the state - action space. The simulation was run during the peak hour. The efficiency of the strategy was measured through delays.

In Table I only a part of the results are shown. The comparison of average delays with the fixed signal control, actuated control, and Q-learning strategy is made.

TABLE I: COMPARISON OF THE AVERAGE DELAYS FOR FIXED CONTROL, ACTUATED CONTROL, AND Q-LEARNING STRATEGY

\begin{tabular}{|c|c|c|}
\hline \multirow{2}{*}{ Run } & \multicolumn{2}{|c|}{$\begin{array}{l}\text { Comparison of average delays for different types of signal } \\
\text { control }\end{array}$} \\
\hline & Type of Control & Average Delay (sec) \\
\hline 278 & Fixed & 0.8312433958 \\
\hline 279 & Fixed & 0,6764412522 \\
\hline 280 & Fixed & 0,9191551175 \\
\hline 281 & Fixed & 0,5908772349 \\
\hline 282 & Fixed & 0,6422748566 \\
\hline 283 & Fixed & 1,1516582966 \\
\hline 284 & Fixed & 0,6924495101 \\
\hline 285 & Fixed & 0,6976267099 \\
\hline 278 & Actuated & 0,232619598507881 \\
\hline 279 & Actuated & 0,175925269722939 \\
\hline 280 & Actuated & 0,192265138030052 \\
\hline 281 & Actuated & 0,1535364985466 \\
\hline 282 & Actuated & 0,159261509776115 \\
\hline 283 & Actuated & 0,249249652028084 \\
\hline 284 & Actuated & 0,15443129837513 \\
\hline 285 & Actuated & 0,163399875164032 \\
\hline 278 & Q - learning & 0,187686145305634 \\
\hline 279 & Q - learning & 0,152055725455284 \\
\hline 280 & Q - learning & 0,182069316506386 \\
\hline 281 & Q - learning & 0,161372631788254 \\
\hline 282 & Q - learning & 0,17327706515789 \\
\hline 283 & Q - learning & 0,191062957048416 \\
\hline 284 & Q - learning & 0,1720094711 \\
\hline 285 & $\mathrm{Q}$ - learning & 0,1137311101 \\
\hline
\end{tabular}

According to the modest simulation results obtained, one can expect some promising and encouraging conclusions. Namely, some reductions of average delays in favor of Q-learning strategy were perceived. However, this hypothesis cannot be scientifically proven at this time because of the small number of iterations and because of the simplicity of the isolated intersection design. 


\section{CONCLUSION}

Although a great number of traffic control strategies has been developed and implemented so far, the field of adaptive signal control has still remained in the focus of many researchers over the past twenty years. The reason behind lies in their numerous limitations such as the uncertainty in predicting future traffic flows, the difficulty in arrival time estimation, the lack of self-adjusting mechanism.

This paper is an attempt to promote the application of adaptive signal control strategy at an isolated intersection by using the technique of artificial intelligence known as reinforcement learning. The research was conducted by using VISSIM micro simulator, and by direct programming of the functions in the simulator.

The adaptive traffic signal control strategy tested on isolated intersection provided encouraging results. The simulation results concerning the average delays indicated that the new approach was more efficient than the one with the fixed and actuated signal control.

Further evaluations of the strategy in different conditions are recommended and different settings of the elements of the $\mathrm{Q}$ learning agent have to be additionally developed.

\section{REFERENCES}

[1] R. S. Sutton and A. G. Barto, Reinforcement Learning - An Introduction. MIT Press, Cambridge, Massachusetts, 1998.

[2] S. Russell and P. Norvi, Artificial Intelligence: A Modern Approach, Prentice Hall, 2009.

[3] Artificial Intelligence in Transportation Information for Application, Transportation Research CIRCULAR, Number E - C 113, Transportation On Research Board of the National Academies, January 2007

[4] B. Abdullah and L. Kitten, "Reinforcement learning: Introduction to theory and potential for transport applications," Can. J. Cave Eng. 30, pp. 981-991, 2003.

[5] B. Abdullah, R. Pringle, and G. J. Karakuls, "Reinforcement Learning for True Adaptive Traffic Signal Control," ASCE Journal of Transportation Engineering, vol. 129, no. 3, pp278-285, 2003.

[6] X.- H. Yu and W. W. Rocker, "Stochastic adaptive control model for traffic signal systems, Transportation Research Part C: Emerging Technologies," vol. 14, Issue 4, pp. 263 - 282, Elsevier, August 2006

[7] Z. Shi, "Principles of Machine Learning," International Academic Publishers, Beijing, 1992.

[8] K. Veljanovska and K. Bombol, "Choosing a new tool for adaptive control strategy design," PhD Research day Research Proceedings, May 9, 2007.

[9] J. C. Spall and D. Chin, "Traffic -Responsive Signal Timing for System-Wide Traffic Control," Transportation Research Part C, vol. 5 , no. 3/4, Elsevier Science Ltd, Great Britain, pp. 153-163,1997.

[10] M. Wiering, Veenen,V, Jelle., J. Vreeken, and A. Koopman, "Intelligent Traffic Light Control," Institute of information and computing sciences, Utrecht University, Technical report UU-CS-2004-029.

[11] D. Roozemond and P. V. D. Veer, "Usability of Intelligent Agent Systems in Urban Traffic Control," Delft University of Technology, Delft, 1999.

[12] A. Meystel and E. Messina, "The Challenge Of Intelligent Systems," Proceedings of the 15th, IEEE International Symposium on Intelligent Control (ISIC 2000), Rio, Patras, GREECE 17-19 July, 2000.

[13] L. Shoufeng, L. Ximin, and Shiqiang, "Q - Learning for Adaptive Traffic Signal Control Based on Delay Minimizations Strategy," IEEE International Conference on Networking Sensing and Control, pp. 687 $-691,2008$

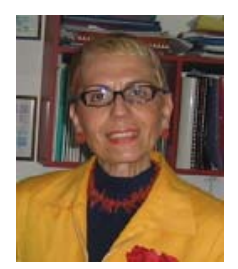

Prof. Kristi Bombol was born in Bitola, Republic of Macedonia in 1957.

Postdoctoral studies (Fulbright grant holder) University of Pennsylvania, Philadelphia, US (1998).

$\mathrm{PhD}$ in Technical Sciences. University in Bitola, Bitola (1996)

Master of Philosophy in Traffic Engineering. University of Belgrade, Belgrade (1989)

Graduated Traffic Engineer. University of Belgrade, Belgrade (1981)

She is a professor at the University in Bitola, at the Department of Traffic and Transport. She has been working for over thirty years now, starting as a young assistant first in 1981 .

Her current research is modern technologies in traffic management and control, sustainable urban traffic systems, accident analysis and prevention. Previous research is vehicle dynamics, biomechanics of traffic accidents.

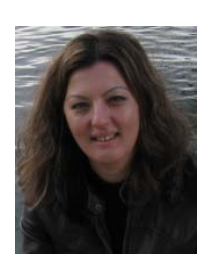

Daniela Koltovska was born in Bitola, Republic of Macedonia in 1975.

Master of Philosophy in Traffic Engineering. University in Bitola, Bitola, (2008)

Graduated Traffic Engineer. University in Bitola. Bitola (2001)

Her present professional occupation is teaching assistant fellow and a PhD student at the University in Bitola, Bitola. Her research interests is Intelligent Traffic Management and Control, Traffic Simulations.

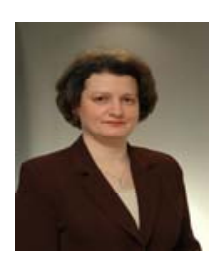

Assist. Prof. Kostandina Veljanovska was born in Bitola, Republic of Macedonia in 1970.

$\mathrm{PhD}$ in Technical Sciences at the University in Bitola, Bitola, Republic of Macedonia (2008)

$\mathrm{MSc}$ in Applied Engineering at the University of Toronto, Toronto, Canada (2003)

MSc in Intelligent Transportation Systems at the University in Bitola, Bitola (2002)

Graduated engineer in Computer Sciences, University in Skopje, Skopje, Republic of Macedonia (1993)

Since 2008 she works as an assistant professor in Computer Science at the

Faculty of Administration and Information Systems Management at the University in Bitola, Bitola.

Her research interests is Artificial Intelligence, Intelligent Systems, Intelligent Transportation Systems, Software Architectures 